\title{
GLOBAL STABILITY OF ENDEMIC EQUILIBRIUM POINT OF BASIC VIRUS INFECTION MODEL WITH APPLICATION TO HBV INFECTION*
}

\author{
Yu ZHENG · Lequan MIN · Yu JI · Yongmei SU • Yang KUANG
}

DOI: $10.1007 / \mathrm{s} 11424-010-8467-0$

Received: 4 January 2009 / Revised: 7 April 2009

(C)The Editorial Office of JSSC \& Springer-Verlag Berlin Heidelberg 2010

\begin{abstract}
In this paper, the authors first show that if $R_{0} \leq 1$, the infection free steady state is globally attractive by using approaches different from those given by Min, et al.(2008). Then the authors prove that if $R_{0}>1$, the endemic steady state is also globally attractive. Finally, based on a patient's clinical HBV DNA data of anti-HBV infection with drug lamivudine, the authors establish an ABVIM. The numerical simulations of the ABVIM are good in agreement with the clinical data.
\end{abstract}

Key words Basic infection reproduction number, clinical data simulation, equilibrium point, global stability, HBV infection.

\section{Introduction}

The basic virus infection model (BVIM) is widely used in the studies of hepatitis B virus (HBV) infection dynamics. The basic infection reproductive number of the BVIM is proportional to the number of all cells of host's organ prior to the infection. This suggests that the BVIM may not be a reasonable model for describing HBV virus infection since it implies that an individual with smaller liver maybe more resistent to virus infection than an individual with a larger one. Min, et al. ${ }^{[1]}$ formulated a standard incidence based model that amended the BVIM (named ABVIM) which corrected this mass action induced model artifact. We have

Yu ZHENG

Information Engineering School, University of Science and Technology Beijing, Beijing 100083, China.

Email: zhengyubeijing@163.com.

Lequan MIN

Mathematics and Physics School, University of Science and Technology Beijing, Beijing 100083, China.

Email: minlequan@sina.com.

Yu JI

Information Engineering School, University of Science and Technology Beijing, Beijing 100083, China.

Email: jylt@163.com.

Yongmei SU

Mathematics and Physics School, University of Science and Technology Beijing, Beijing 100083, China.

Email: suym71@163.com.

Yang KUANG

Department of Mathematics and Statistics, Arizona State University, Temp AZ 85287-1804, USA.

Email: kuang@asu.edu.

*This research is supported by the National Natural Science Foundations of China under Grant No. 60674059, the 11th 5-Year Plan Key Research Project of China under Grant No. 2004BA721A03, the China National Key Special Project for the Preventions and Cures of Important Infectious Diseases under Grant No. 2008ZX10005006. 
proved that if its basic infection reproductive number $R_{0}<1$, then the infection free steady state is globally attractive.

More than 400 million people worldwide have chronic hepatitis B virus (HBV) infection. Effective treatment of chronic HBV infection patients can prevent progression of chronic hepatitis $\mathrm{B}(\mathrm{CHB})$ to cirrhosis, hepatocellular carcinoma, and death. In view of the need for long-term treatment, the choices of durations and endpoints of therapy are important and difficult issues.

The study of anti-HBV infection treatment may benefit from the use of mathematical modeling. Several models have been introduced for understand the HBV dynamics ${ }^{[2-5]}$. However, the complex characteristics of human HBV make theoretical researchers difficult to determine the specific kinetic parameters of HBV infection, immune responses, and development of liver disease $^{[6]}$.

Nowak and May ${ }^{[2]}$, and Zeuzem, et al. ${ }^{[5]}$ proposed a mathematical model named BVIM to describe the hepatitis virus infection. The BVIM with three variables takes the form of

$$
\left\{\begin{array}{l}
\dot{x}=\lambda-d x-\beta v x \\
\dot{y}=\beta v x-a y \\
\dot{v}=k y-u v
\end{array}\right.
$$

where $x, y$ and $v$ are numbers of uninfected (susceptible) cells, infected cells, and free virus, respectively. Uninfected cells are assumed to be produced at the constant rate $\lambda$, die at the rate of $d x$, and become infected at the rate of $\beta v x . \beta$ is a (questionable) rate constant describing the infection process. Infected cells are thus produced at the rate of $\beta v x$ and are assumed to die at the rate $a y$. Free virions are assumed to be produced from infected cells at the rate of $k y$ and are removed at the rate of $u v$. This model is used for studying the dynamic of infectious agents such as hepatitis B, C and HIV virus ${ }^{[2-3,5-6]}$. BVIM has a basic infection reproductive number of $R_{0}^{*}=\frac{\lambda \beta k}{a d \mu}$. If $R_{0}^{*}>1$, then the BVIM has two steady states, the infection free steady state $E_{f}$ and the endemic steady state $E^{*}$ :

$$
\begin{aligned}
E_{f} & =\left(\frac{\lambda}{d}, 0,0\right), \\
E^{*} & =\left(\frac{a u}{\beta k}, \frac{\lambda}{a}\left(1-\frac{1}{R_{0}^{*}}\right), \frac{d}{\beta}\left(R_{0}^{*}-1\right)\right) .
\end{aligned}
$$

It is well-known that if a basic reproductive number $R_{0}^{*}<1, E_{f}$ is locally asymptotically stable and $E^{*}$ does not exist. Leenheer and Smith ${ }^{[7]}$ studied the global attractive properties of these steady states.

Observe that the basic infection reproductive number $R_{0}^{*}$ is proportional to $\lambda / d$ which stands for the number of total cells of the liver. It suggests that a person with a smaller liver maybe more resistent to virus infection than a person with a larger one. Consequently, the practical meaning of $R_{0}^{*}$ is biologically questionable, the BVIM may not be a reasonable model for interpreting HBV virus infection.

In [1], we proposed an amended basic HBV virus model (ABVIM):

$$
\left\{\begin{array}{l}
\dot{x}=\lambda-d x-\frac{\beta v x}{x+y} \\
\dot{y}=\frac{\beta v x}{x+y}-a y \\
\dot{v}=k y-u v
\end{array}\right.
$$

where the meanings of the variables $x, y, v$ and the parameters $\lambda, d, a, k, u$ are the same as those of the BVIM. However, here $\beta$ has a clear biological meaning which is the maximum infection 
rate of a virus. Its basic reproductive number $R_{0}=\beta k / a u$, which is not dependent on the total number of cells of the individual's liver. Theoretically, the ABVIM seems to be more reasonable. As an application of the ABVIM, it can interpret the viral dynamics of the anti-HBV infection 48-week lamivudine therapy and 24-week treatment-free follow up (see [8]). Our previous paper proved that if its basic infection reproductive number $R_{0}<1$, then every positive solution will converge to the infection free steady state. An extension of this model with a time delay in infection was presented and studied in [9].

The rest of this paper is organized as follows. Section 2 states the global stability analysis of equilibrium points. An application to HBV infection dynamics is given in Section 3. Finally, concluding remarks are addressed in Section 4.

\section{Global Stability of Equilibrium Points}

If $R_{0} \geq 1$, the ABVIM also has two steady states:

$$
\begin{aligned}
& E_{f}=\left(\frac{\lambda}{d}, 0,0\right) \\
& E^{*}=\left(\frac{\lambda}{d+a\left(R_{0}-1\right)}, \frac{\lambda\left(R_{0}-1\right)}{d+a\left(R_{0}-1\right)}, \frac{\lambda k\left(R_{0}-1\right)}{u\left[d+a\left(R_{0}-1\right)\right]}\right),
\end{aligned}
$$

representing the disease free steady state and the endemic steady state, respectively. Observe that a biologically meaningful $E^{*}$ (meaning its component must be nonnegative) does not exist if $R_{0}<1$ and it becomes $E_{f}$ when $R_{0}=1$.

It is easy to show that the solution with initial condition $x(0)>0, y(0)>0, v(0)>0$ will have all its component positive for $t>0$. First, using a different approach from that in [1], we show the globally stable property of the disease free steady state $E_{f}$.

Theorem 1 There is an $M>0$ such that, for any positive solution $(x(t), y(t), v(t))$ of Equation (2), we have

$$
x(t)<M, y(t)<M, v(t)<M, \text { for large enough } t .
$$

Proof Let

$$
V_{1}(t)=x(t)+y(t)
$$

Calculating the derivative along the solutions of Equation (2) gives

$$
\dot{V}_{1}(t)=\lambda-d x-a y \leq \lambda-\min (a, d) V_{1}(t) .
$$

Denote $m=\min (a, d)$, it follows that

$$
\dot{V}_{1}(t)+m V_{1}(t) \leq \lambda .
$$

Further,

$$
V_{1}(t) \leq \frac{\lambda}{m}+\left(V_{1}(0)-\frac{\lambda}{m}\right) \mathrm{e}^{-m t}
$$

Hence, there exists $t_{1}>0$ and $M_{1}>0$ such that $V_{1}(t)<M_{1}$ for $t>t_{1}$. So $x(t)$ and $y(t)$ are bounded. On the other hand, from the third equation of system (2), we obtain

$$
\dot{v}(t)=k y-u v \leq k M_{1}-u v .
$$


Similar to the argument on $V_{1}(t)$, we can conclude that $v(t)$ is also ultimately bounded.

Theorem 2 If $R_{0}<1$, then $E_{f}$ is globally asymptotically stable.

Proof From the last two equations of system (2), for $t>t_{1}$, we have

$$
\left\{\begin{array}{l}
\dot{y}=\frac{\beta v x}{x+y}-a y \leq \beta v-a y, \\
\dot{v}=k y-u v
\end{array}\right.
$$

Consider the comparison equations:

$$
\left\{\begin{array}{l}
\dot{z_{1}}=\beta z_{2}-a z_{1} \\
\dot{z_{2}}=k z_{1}-u z_{2} .
\end{array}\right.
$$

Since $R_{0}<1, \beta k<a u$. It is easy to show system (3) has two negative eigenvalues. For any solution of system (3) with nonnegative values, we have

$$
\lim _{t \rightarrow+\infty} z_{i}(t)=0, i=1,2 .
$$

Let $0<y(0)<z_{1}(0), 0<v(0)<z_{2}(0)$, by the comparison theorem, it follows that $y(t)<$ $z_{1}(t), v(t)<z_{2}(t)$ for all $t>t_{1}$. Therefore,

$$
\lim _{t \rightarrow+\infty} y(t)=0, \quad \lim _{t \rightarrow+\infty} v(t)=0 .
$$

From the first equation of system (2), we can get

$$
\lambda-d x-\beta v \leq \dot{x}(t) \leq \lambda-d x .
$$

This shows that there exists $t_{2}>0$, such that $t>t_{2}$,

$$
\lim _{t \rightarrow+\infty} x(t)=\frac{\lambda}{d} .
$$

Let $t_{0}=\max \left(t_{1}, t_{2}\right)$, for all $t>t_{0}$,

$$
\lim _{t \rightarrow+\infty} x(t)=\frac{\lambda}{d}, \quad \lim _{t \rightarrow+\infty} y(t)=0, \quad \lim _{t \rightarrow+\infty} v(t)=0 .
$$

This completes the proof.

Theorem 3 If $R_{0}=1$, then $E_{f}$ is globally asymptotically stable.

Proof The solution to the third equation of system (2) has the form

$$
v(t)=v(0) \mathrm{e}^{-u t}+\frac{\int_{0}^{t} k y(s) \mathrm{e}^{u s} d s}{\mathrm{e}^{u t}},
$$

thus,

$$
\left|v(t)-\frac{k y(t)}{u}\right| \rightarrow 0, \quad t \rightarrow+\infty .
$$

Hence, for any $\varepsilon>0$ small enough, there exists $t_{3}>0$, for all $t>t_{3}$ such that

$$
\frac{d y}{d t}=\frac{\beta v x}{x+y}-a y<\beta \varepsilon+\frac{\left(\beta \frac{k}{u}-a\right) x y}{x+y}-\frac{a y^{2}}{x+y} .
$$


Since $\varepsilon>0$ is small enough and $R_{0}=\frac{\beta k}{a u}=1$, the above inequality can be written as

$$
\frac{\dot{y}}{y^{2}} \leq-\frac{a}{M_{1}}
$$

which implies

$$
\lim _{t \rightarrow+\infty} y(t)=0
$$

(4) and (5) gives

$$
\lim _{t \rightarrow+\infty} v(t)=0
$$

From the first equation of system (2), we obtain

$$
\lambda-d x-\beta v \leq \dot{x}(t) \leq \lambda-d x .
$$

From (5)-(7), we have

$$
\lim _{t \rightarrow+\infty} x(t)=\frac{\lambda}{d} .
$$

This completes the proof of Theorem 3 .

In order to show the globally stable property of the endemic steady state $E^{*}$, we need first to show the following theorem.

Theorem 4 If $R_{0}>1$, the system (2) is uniformly persistent.

Proof By the Lemma 3.5 given in [7], we only need to show that $E_{f}$ is a weak repeller for $X_{1}=\operatorname{int}\left(\mathbb{R}_{+}^{3}\right)$, that is, for every solution starting in $X_{0} \in X_{1}$, we have

$$
\lim _{t \rightarrow+\infty} \sup d\left(\boldsymbol{X}\left(t, \boldsymbol{X}_{0}\right), E_{f}\right)>0
$$

where $d(\boldsymbol{X}, A)$ denotes the distance between the vector $\boldsymbol{X}=(x(t), y(t), v(t))$ to the set $A \subset \mathbb{R}^{3}$.

In fact, if $E_{f}$ is not a weak repeller for $X_{1}=\operatorname{int}\left(R_{+}^{3}\right)$, then (8) does not hold. It follows that there is a positive orbit $(x(t), y(t), v(t))$ of system $(2)$ such that

$$
\lim _{t \rightarrow+\infty} x(t)=\frac{\lambda}{d}, \quad \lim _{t \rightarrow+\infty} y(t)=0, \quad \lim _{t \rightarrow+\infty} v(t)=0 .
$$

Since $R_{0}>1$, we can choose $\varepsilon>0$ small enough such that

$$
\frac{\beta k}{a u} \frac{\frac{\lambda}{d}-\varepsilon}{\frac{\lambda}{d}+2 \varepsilon}>1 .
$$

For this $\varepsilon$ and (9), we can select $t_{0}>0$ large enough such that if $t>t_{0}$ then

$$
\frac{\lambda}{d}-\varepsilon<x(t)<\frac{\lambda}{d}+\varepsilon, \quad y(t)<\varepsilon, \quad v(t)<\varepsilon .
$$

From the last two equations of system (2), for $t>t_{0}$, we obtain

$$
\left\{\begin{array}{l}
\dot{y}=\frac{\beta v x}{x+y}-a y \geq \frac{\frac{\lambda}{d}-\varepsilon}{\frac{\lambda}{d}+2 \varepsilon} \beta v-a y \\
\dot{v}=k y-u v
\end{array}\right.
$$


Consider the comparison equations:

$$
\left\{\begin{array}{l}
\dot{z_{1}}=\frac{\frac{\lambda}{d}-\varepsilon}{\frac{\lambda}{d}+2 \varepsilon} \beta z_{2}-a z_{1}, \\
\dot{z}_{2}=k z_{1}-u z_{2}
\end{array}\right.
$$

the matrix defined by

$$
J=\left(\begin{array}{cc}
-a & \frac{\frac{\lambda}{d}-\varepsilon}{\frac{\lambda}{d}+2 \varepsilon} \beta \\
k & -u
\end{array}\right) .
$$

Since $J$ is a matrix with positive off-diagonal element, the Perron-Frobenius theorem implies that there is positive eigenvector $v=\left(v_{1}, v_{2}\right)$ for the maximum eigenvalue of $J$. Moreover,

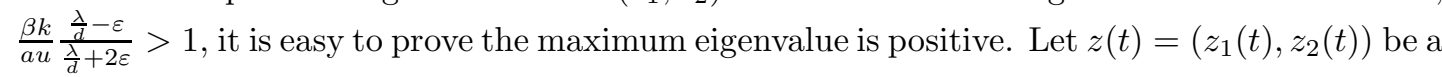
solution of system (10) through $\left(l v_{1}\left(t_{0}\right), l v_{2}\left(t_{0}\right)\right)$, where $l>0$ satisfies $l v_{1}\left(t_{0}\right)<y\left(t_{0}\right), l v_{2}\left(t_{0}\right)<$ $v\left(t_{0}\right)$. It follows that

$$
\lim _{t \rightarrow+\infty} z_{i}(t)=+\infty, i=1,2 .
$$

By the comparison theorem,

$$
\lim _{t \rightarrow+\infty} y(t)=+\infty, \quad \lim _{t \rightarrow+\infty} v(t)=+\infty,
$$

contradicting the boundedness of the positive solution of system (2). Thus, no positive orbit of system (2) tends to $\left(\frac{\lambda}{d}, 0,0\right)$ as $t$ tends to infinity. This shows that system (2) is uniformly persistent.

Now, let us define

$$
D=\left\{(x, y, v): 0 \leq x(t) \leq \frac{\lambda}{d}, 0 \leq y(t)<M, 0 \leq v(t)<M\right\} .
$$

We can get that the system (2) is competitive in $D$, with respect to the partial order defined by the orthant

$$
K=\left\{(x, y, v) \in R^{3}: x(t) \geq 0, y(t) \geq 0, v(t) \geq 0\right\} .
$$

By means of the following theorem, we show that the globally stable property of $E^{*}$ when $R_{0}>1$.

Theorem $5^{[10]}$ Assume that $D$ is convex and bounded. Suppose that system

$$
\dot{X}=F(X), X \in D
$$

is competitive and permanent, and has the property of stability of periodic orbit. If $\bar{X}_{0}$ is the only equilibrium point in $\operatorname{int}(D)$, and if it is locally asymptotically stable, then it is globally asymptotically stable in $\operatorname{int}(D)$.

Theorem 6 Suppose that $R_{0}>1$, then $E^{*}$ is globally asymptotically stable with respect to initial conditions which are not located on the $x$ axis.

Proof Similar to the arguments stated in [11], we know that the only possible omega limit sets of the solutions of system (2) are $E^{*}$ or nontrivial periodic orbits:

1) If an omega limit set does contain $E^{*}$. We establish the claim.

2) If system (2) possesses a nontrivial periodic solution. Similar to the arguments stated in [12], we shall show this solution must be asymptotically orbitally stable. Assume that 
$p(t)=(x(t), y(t), v(t))$ is a periodic solution containing in $\operatorname{int}(D)$. By the criterion given in [12], we only need to prove that the system

$$
\dot{W}(t)=\left(D F^{[2]}(p(t))\right) W(t)
$$

is asymptotically stable, where $D F^{[2]}$ is the second additive compound matrix of the Jacobian $J$.

The Jacibian of system (2) and its second additive compound matrix have the forms

$$
\begin{gathered}
J=\left(\begin{array}{ccc}
-d-\frac{\beta v y}{(x+y)^{2}} & \frac{\beta v x}{(x+y)^{2}} & -\frac{\beta x}{x+y} \\
\frac{\beta v y}{(x+y)^{2}} & -\frac{\beta v x}{(x+y)^{2}}-a & \frac{\beta x}{x+y} \\
0 & k & -u
\end{array}\right), \\
D F^{[2]}=\left(\begin{array}{ccc}
-d-a-\frac{\beta v}{x+y} & \frac{\beta x}{x+y} & \frac{\beta x}{x+y} \\
k & -d-u-\frac{\beta v y}{(x+y)^{2}} & \frac{\beta v x}{(x+y)^{2}} \\
0 & \frac{\beta v y}{(x+y)^{2}} & -a-u-\frac{\beta v x}{(x+y)^{2}}
\end{array}\right) .
\end{gathered}
$$

Substituting $p(t)$ into (11) gives

$$
\begin{aligned}
& \dot{W}_{1}=\left(-d-a-\frac{\beta v}{x+y}\right) W_{1}+\frac{\beta x}{x+y}\left(W_{2}+W_{3}\right), \\
& \dot{W}_{2}=k W_{1}+\left(-d-u-\frac{\beta v y}{(x+y)^{2}}\right) W_{2}+\frac{\beta v x}{(x+y)^{2}} W_{3}, \\
& \dot{W}_{3}=\frac{\beta v y}{(x+y)^{2}} W_{2}+\left(-a-u-\frac{\beta v x}{(x+y)^{2}}\right) W_{3} .
\end{aligned}
$$

We will show that the function

$$
V\left(W_{1}, W_{2}, W_{3}\right)=\sup \left\{\left|W_{1}\right|, \frac{y(t)}{v(t)}\left(\left|W_{2}\right|+\left|W_{3}\right|\right)\right\}
$$

is a Lyapunov function for system (11). We have

$$
\begin{aligned}
& D_{+}\left|W_{1}\right| \leq\left(-d-a-\frac{\beta v}{x+y}\right)\left|W_{1}\right|+\frac{\beta x}{x+y}\left(\left|W_{2}\right|+\left|W_{3}\right|\right), \\
& D_{+}\left|W_{2}\right| \leq k\left|W_{1}\right|+\left(-d-u-\frac{\beta v y}{(x+y)^{2}}\right)\left|W_{2}\right|+\frac{\beta v x}{(x+y)^{2}}\left|W_{3}\right|, \\
& D_{+}\left|W_{3}\right| \leq \frac{\beta v y}{(x+y)^{2}}\left|W_{2}\right|+\left(-a-u-\frac{\beta v x}{(x+y)^{2}}\right)\left|W_{3}\right|,
\end{aligned}
$$

where $D_{+} V(t)$ is the right derivative of $V$, and

$$
\begin{aligned}
D_{+} \frac{y(t)}{v(t)}\left(\left|W_{2}\right|+\left|W_{3}\right|\right) & =\left(\frac{\dot{y}(t)}{y(t)}-\frac{\dot{v}(t)}{v(t)}\right) \frac{y(t)}{v(t)}\left(\left|W_{2}\right|+\left|W_{3}\right|\right)+\frac{y(t)}{v(t)} D_{+}\left(\left|W_{2}\right|+\left|W_{3}\right|\right) \\
& \leq k \frac{y(t)}{v(t)}\left|W_{1}\right|+\frac{y(t)}{v(t)}\left(\frac{\dot{y}(t)}{y(t)}-\frac{\dot{v}(t)}{v(t)}-u-\min (a, d)\right)\left(\left|W_{2}\right|+\left|W_{3}\right|\right) .
\end{aligned}
$$


Thus, we obtain that

$$
D_{+} V(t) \leq \sup \left\{g_{1}(t), g_{2}(t)\right\} V(t)
$$

where

$$
\begin{aligned}
& g_{1}(t)=-d-a-\frac{\beta v}{x+y}+\frac{v}{y} \frac{\beta x}{x+y}=-d-\frac{\beta v}{x+y}+\frac{\dot{y}(t)}{y(t)} \\
& g_{2}(t)=k \frac{v}{y}+\frac{\dot{y}(t)}{y(t)}-\frac{\dot{v}(t)}{v(t)}-u-\min (a, d)=\frac{\dot{y}(t)}{y(t)}-\min (a, d) .
\end{aligned}
$$

Hence, $g_{1}(t)<g_{2}(t)$. We have

$$
V(t) \leq V(0) y(t) \mathrm{e}^{-\min (a, d) t},
$$

which implies that $V(t) \rightarrow 0$ as $t \rightarrow+\infty$. It turns out that

$$
\left(W_{1}(t), W_{2}(t), W_{3}(t)\right) \rightarrow 0
$$

as $t \rightarrow+\infty$. This implies that the linear system Equation (11) is asymptotically stable and therefore $p(t)$ is asymptotically orbitally stable. This completes the proof.

\section{An Application to HBV Infection Dynamics}

In this section, we simulate the dynamics of the antiviral treatment of chronic HBV infection with drug lamivadine reported by Nowak, et al. ${ }^{[13]}$. The study comprised 24-week treatment and 24-week treatment-free follow-up.

We choose the patient 2's plasma HBV DNA data shown in Figure 1 (B) given in [13]. The patient's plasma HBV DNA levels decline rapidly, in response to drug treatment and virus resurges as soon as the drug is withdrawn (see Table 1 and Figure 1). The patient's ALT levels are normal during the therapy ${ }^{[13]}$. This may implies that the patient's immune system was not activated.

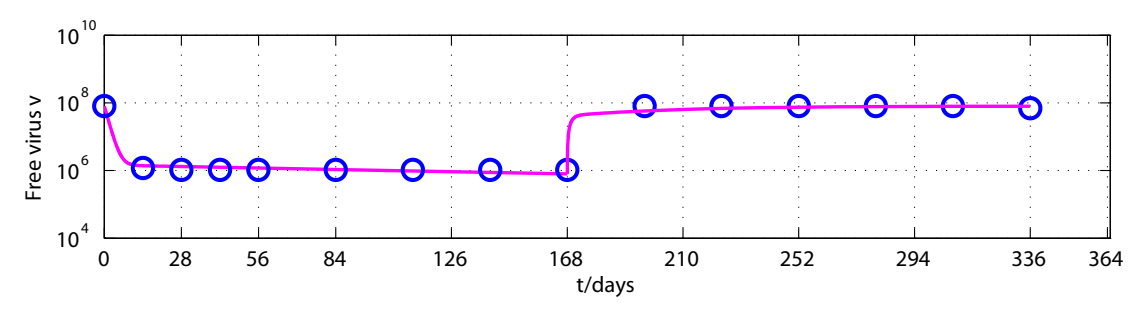

Figure 1 The dynamic simulation (solid lines) of the treatment model in which the clinical data are marked by dots. When $R_{0}=0.162$, virus declines in response to drug treatment and the free equilibrium $E_{f}$ is globally asymptotically stable. When $R_{0}=9$, virus resurges as soon as the drug is withdrawn, the endemic equilibrium $E^{*}$ is globally asymptotically stable

Consequently, we can assume that during the lamivudine drug treatment, the dynamic model of the patient's anti-HBV infection therapy is described by the following ABVIM ${ }^{[1]}$ 


$$
\begin{aligned}
& \frac{d x}{d t}=\lambda-d x-(1-m) \frac{\beta v x}{x+y}, \\
& \frac{d y}{d t}=(1-m) \frac{\beta v x}{x+y}-a y, \\
& \frac{d v}{d t}=(1-n) k y-u v,
\end{aligned}
$$

where $m, n$ are the efficacy of the therapy. Before the drug therapy, assume that $m=n=0$, and the patient is in the stable state $E^{*}$. Therefore,

$$
k=\frac{u v(0)\left[d+a\left(R_{0}-1\right)\right]}{\lambda\left(R_{0}-1\right)}, \quad \beta=\frac{a u R_{0}}{k} .
$$

The followings are detailed steps involved in the estimations of model parameters.

1) A human liver contains about $2 \times 10^{11}$ hepatocytes ${ }^{[2]}$. A patient has about total $3000 \mathrm{ml}$ plasma. Usually, tested virus qualities are in copies/ml. Consequently, we can assume that

$$
\frac{\lambda}{d} \approx \frac{2 \times 10^{11}}{3000} .
$$

2) Since the half-life of a hepatocyte is about half a year, we can assume that

$$
d=-\frac{\ln (0.5)}{183} \approx 0.00379
$$

3) We select that $u=0.67^{[2]}$, which is equivalent to assume that the half life of a virus is about one day.

4) Assume that before the lamivudine treatment, the patient is in the stable virus persist infection state, that is,

$$
(\bar{x}, \bar{y}, \bar{v})=E^{*}=\left(\frac{\lambda}{d+a\left(R_{0}-1\right)}, \frac{\lambda\left(R_{0}-1\right)}{d+a\left(R_{0}-1\right)}, \frac{\lambda k\left(R_{0}-1\right)}{u\left[d+a\left(R_{0}-1\right)\right]}\right) .
$$

5) Based on the clinical data and numerical simulation, we can select the parameters as follows:

$$
\left\{d, a, u, R_{0}, m, n\right\}=\left\{3.79 \times 10^{-3}, 3.79 \times 10^{-3}, 0.67,9,0,0.982\right\} .
$$

The basic reproductive number $R_{0}=9$ is the one before the therapy (i.e., $m=n=0$ ). $R_{0}$ will be reduced to 0.162 during the treatment. After the lamivudine treatment stop, the patient is assumed to return to the state before the therapy, that is, $m=n=0$. Taken the endemic steady state $E^{*}$ as the initial condition, the numerical simulation is shown in Figure 1. The simulated data are given in Table 1. Observe from Figure 1 that after the onset of therapy viral levels decline rapidly, but as soon as the drug is withdrawn, virus level returns rapidly. Figure 1 indicates that the model simulation agrees well with the clinical data reported in [13].

Table 1 Rapid decline in plasma virus: mean HBV DNA levels $\left(\log _{10}\right.$ copies $\left./ \mathrm{ml}\right)$ in response to the therapy, and the virus level returning rapidly after the treatment was stopped

\begin{tabular}{ccccccccc}
\hline Week & 0 & 2 & 4 & 6 & 8 & 12 & 16 & 20 \\
\hline Virus load & 7.90 & 6.08 & 6.02 & 6.03 & 6.02 & 6.03 & 6.02 & 6.03 \\
Simulation & 7.90 & 6.14 & 6.11 & 6.10 & 6.07 & 6.03 & 5.99 & 5.94 \\
\hline \hline Week & 24 & 28 & 32 & 36 & 40 & 44 & 48 & - \\
\hline Virus load & 6.02 & 7.90 & 7.90 & 7.90 & 7.90 & 7.90 & 7.85 & - \\
Simulation & 5.90 & 7.76 & 7.83 & 7.87 & 7.89 & 7.90 & 7.90 & - \\
\hline
\end{tabular}




\section{Concluding Remarks}

The widely used basic virus infection model ${ }^{[2]}$ has been amended ${ }^{[1,9]}$ because its reproductive number $R_{0}$ includes a questionable term $\lambda / d$ representing the number of total cells of the organ to be infected. The amended basic virus infection model (ABVIM) has a more reasonable reproductive number $R_{0}=\beta k / a u$. It has been shown that if $R_{0}<1$ the virus free steady state of the ABVIM is globally attractive ${ }^{[1]}$. This means that a person with $R_{0}<1$ cannot be infected by virus forever.

This paper first proves, using a different approach, that the free steady state $E_{f}$ is globally attractive if $R_{0} \leq 1$. Then shows that if $R_{0}>1$, the endemic steady state $E^{*}$ of ABVIM is also globally attractive. This means that a person with $R_{0}>1$ will be very difficult to prevent to be infected. Consequently, HBV vaccines may be the first line choice for preventing HBV infection.

Theorem 6 also implies that if a patient's $R_{0}>1$, and drug anti-virus therapy cannot activate the patient's immune response, then the anti-virus treatment cannot stop until all virus has been cleared. A practical example illustrates that our theoretical and numerical results are able to provide helps for understanding HBV infection and anti-HBV infection therapy.

\section{References}

[1] L. Q. Min, Y. M. Su, and Y. Kuang, Mathematical analysis of a basic model of virus infection with application to HBV infection, Rocky Mountain J. Math., 2008, 38(5): 1573-1584.

[2] M. A. Nowak and R. M. May, Viral Dynamics, Oxford University Press, Oxford, 2000.

[3] A. Perelson, A. Neumann, M. Markowitz, et al., HIV-1 dynamics in vivo: Virion clearance rate, infected cell life-span, and viral generation time, Science, 271(5255): 1582-1586.

[4] A. Neumann, N. Lam, H. Dahari, et al., Hepatitis C viral dynamics in vivo and antiviral efficacy of the interferon-therapy, Science, 1998, 282(5386): 103-107.

[5] S. Zeuzem, J. M. Schmidt, J. H. Lee, et al., Effect of interferon alfa on the dynamics of hepatitis C virus turnover in vivo, J. Hepatology, 1996, 23(2): 366-371.

[6] M. A. Feitelson and J. D. Larkin, New animal models for hepatitis B and C, Ilar J., 2001, 42(2): $127-138$.

[7] P. D. Leenheer and H. L. Smith, Virus dynamics: A global analysis, SIAM J. Appl. Math., 2003, 63(4): 1313-1327.

[8] G. K. Lau, T. Piratvisuth, K. X. Luo, et al., Peginterferon Alfa-2a, Lamivudine, and the Combination for HBeAg-Positive Chronic Hepatitis B, N. Engl. J. Med., 2005, 352(26): 2682-2695.

[9] S. A. Gourley, Y. Kuang, and J. D. Nagy, Dynamics of a delay differential model of hepatitis B virus, J. Biological Dynamics, 2008, 2(2): 140-153.

[10] X. Y. Song and A. Neumann, Global stability and periodic solution of the viral dynamics, J. Math. Anal. Appl., 2007, 329(1): 281-297.

[11] H. L. Smith, Monotone Dynamical Systems, AMS, Providence, RI, 1995.

[12] J. S. Muldowney, Compound matrices and ordinary differential equations, Rocky Mountain J. Math., 1990, 20(4): 857-872.

[13] M. A. Nowak, S. Bonhoeffer, A. M. Hill, et al., Viral dynamics in hepatitis B virus infection, Proc. Natl. Acad. Sci. USA, 1996, 93(9): 4398-4402. 